\title{
A METHOD FOR THE ESTIMATION OF THE RISK PREMIUMS IN STOP LOSS REINSURANCE
}

\section{Carl Philipson, Sweden}

1. The reinsurance to be treated in this note shall cover the excess over a certain limit $Q$ of the total amount of claims for each accounting period paid by the ceding company. Regardless of the rule for the determination of the limit $Q$ such a reinsurance shall in this context be called stop loss reinsurance.

Generally, such a reinsurance is called either stop loss or excess of loss reinsurance depending on the rule for the determination of $Q$. The use of the term stop loss regardless of this rule is preferred here in order to avoid confusion with an excess of loss reinsurance which refers to the excess of the amount of one or more claims caused by an individual event.

2. The total amount of claims paid by the ceding company $c$ during the period $t$ shall be denoted ${ }_{c} X_{t}$. This random function constitutes for a fixed value of $c$ a stochastic process with the discontinuous parameter $t>0$.

(It may be remarked that for a fixed pair of values of $c$ and $t$ ${ }_{c} X_{t}$ can be regarded as a particular value of a sample function pertaining to the process of the continuous paramater $\tau$ generally considered in the collective theory of risk to which the total amount of claims up to time $\tau$ is attached, in this case $0<\tau \leqslant \mathrm{I} .{ }_{c} X_{t}$ can also be considered as the increments during the time interval $t$ of the total amount of the claims up to $\tau$.)

The process constituted by ${ }_{c} X_{t}$ is, generally, non-stationary. In fact, the value of ${ }_{c} X_{t}$ for a fixed pair of values of $c$ and $t$ depends inter alia on the distribution of the (risk) sums insured over amounts and risk groups which, in general, is dependent on time. Furthermore ${ }_{c} X_{t}$ is generated by the ordinary risk process as described above. In this process the probability of one claim in an infinitesimally small interval of $\tau$ depends, as a rule (in general insurance), not only on time passed but also on the number of risk situations 
which have occurred before the interval (the Markoff property). Also the conditioned distribution for the amount of one claim (provided such a claim has incurred) can depend on time.

3. In order to elucidate the statements of ${ }_{c} X_{t}$ madein section 2 here above we will now analyze this function for a fixed pair of values of $c$ and $t$. Consider, for this purpose, a division of the policies written by the ceding company $c$ in force during any part of the period $t$ into a number of elementary groups denoted by the index vector $i$. This vector shall include the measures of all factors governing the risk (for non-measurable qualitative factors each quality shall be associated with a rational number which shall assign the measure). Inter alia the magnitude of the (risk) sum insured shall be included in the vector, eventually only for certain intervals. For such an elementary group $i,{ }_{c} X_{i t}$ shall denote the total amount of claims paid for the group during the period $t$, ${ }_{c} R_{i t}$ the (risk) sum insured under risk for the period and $z_{i t}$ the quotient between the first-mentioned quantities, which quotient is considered independent of $c$.-For such insurance forms as have no defined sum insured, e.g. third party liability, ${ }_{c} R_{i t}$ shall be interpreted as the number of policies under risk. By definition we have, thus, for every fixed pair of values for $c$ and $t$

$$
{ }_{c} X_{t}=\Sigma_{c} R_{i i} z_{i t},
$$

where the summation shall refer to all possible values of $i$.

4. As a basic assumption for all the subsequent developments we suppose that $z_{i t}$ can be approximated by a product $p_{i t} y_{i t}$ where $p_{i t}$ is non-stationary and $y_{i t}$ is attached to a stationary process with ergodic properties described in the subsequent section. This is in conformance with the factor analysis as treated by Almer for Motor Insurance (Trans. XVth. Int. Congr. Act., I957, II, pp. 344-349).

Consequently we may write

$$
{ }_{c} X_{t}=\Sigma p_{i t \cdot c} R_{i t} y_{i}=\Sigma{ }_{c} R_{i t}^{\prime} y_{i}
$$

with the same interpretation of the summation as in ( $\mathrm{I}$ ).

5. In order to deduce the risk premium of the reinsurance defined in section $\mathbf{I}$ we will in this section impose restrictions by making 
particular assumptions regarding the ceding company, these are then removed in sections 6 and 7 .

Consider a ceding company with only one elementary group with the (risk) sum insured under risk independent of $t$ and $p_{i t}=\mathrm{I}$ independently of $t$. Thus, $z_{i t}=y_{i}$, where the index $i$ can be omitted for all values of $t . y_{t}$ is attached to an ergodic process. Such a process might be defined as a stationary process for which the distribution function of a given realization belongs to a system of such functions which coincides with the system defining the basic probabilities of the process. This property of the process is a necessary and sufficient condition for another property often used for the definition of ergodicity. The last mentioned property involves that, for any function of $y_{t}$ with finite mean square, the phase average for a given realization exists and is, with probability $I$, equal to the corresponding space average of the function. The distribution function of the standardized variable $u=\frac{y-m}{\sigma}, m, \sigma$ denoting the mean and standard deviation of $y$ (the indices $i$ are omitted), can-under fairly general conditions-be approximated by a distribution function with existing first derivative such that it might be asymptotically expanded in the Edgeworth's series $\varphi(u)+\sum_{v=3}^{6} a_{v} \varphi^{(v)}(u)$, where $\varphi(u)$ is the normal density function and $a_{3}=-\frac{\gamma_{1}}{3 !} ; a_{4}=\frac{\gamma_{2}}{4 !} ; a_{5}=0, a_{6}=\frac{a^{2}}{72} ; \gamma_{1}, \gamma_{2}$ being the skewness and excess respectively of $y$ (the indices $i$ are omitted). Writing $q$ for the limit per unit (risk) sum insured (for the reinsurance defined in (I)), the risk premium rate, $r$, i.e. the risk premium in the same units, can be defined as the phase average for a given realization of $y-q$ over the subspace defined by $y>q$.

Under these provisional assumptions we find, after some calculation, the following approximate expression for the risk premium rate $r=r(m, \sigma, q)$ writing $\Phi(v), \varphi(v)$ for the normal distribution, and density function respectively and $v_{q}=\frac{q-m}{\sigma}$, omitting the indices $i$

$$
r(m, \sigma, q)=\sigma\left[\varphi\left(v_{q}\right)-v_{q}\left(\mathrm{I}-\Phi\left(v_{q}\right)+\sum_{\nu=3}^{6} a_{\nu} \varphi^{(v-2)}\left(v_{q}\right)\right]\right.
$$


With respect to the rule for the determination of $Q$ this formula is quite general. It has been shown elsewhere, Skand. Akt. Tidskr. I958, pp. 7I-92. that material advantages will be obtained if one assumes $q=m+k \sigma$, where $k$ is a constant independent of any statistics and independent of $i$. In this case a constant $K=\varphi(k)$ - $k(\mathrm{I}-\Phi(k))$ can be substituted for the first two terms in brackets in (3).

If the estimate of the risk premiums should be based on (3) as it stands it is necessary to estimate not only $\sigma$ but also the 3 rd and the 4th semi-invariants in $a_{y}$, from a sample. Such a sample can, however, be used also for finding a direct estimate of $r$ based on the sample mean of indemnities. If such a direct estimate for $q=m+k \sigma$ is equated to $\varepsilon \sigma . K$, and $\varepsilon$ determined from the equation, the risk premium rate can be written $\varepsilon \sigma K$. This method can be further modified by equating the direct estimate for $q^{\prime}=$ $m+\delta k \sigma$ to $\delta \sigma K$ for the determination of $\delta$. It can be shown that, generally, $\delta$ is materially less than $\varepsilon$. We may, thus, write the risk premium rate for $q^{\prime}=m+k \sigma^{\prime}$, where $\sigma^{\prime}=\delta \sigma$ may be called the modified standard deviation and

$$
r\left(m, \sigma^{\prime}, q^{\prime}\right)=\sigma^{\prime} . K
$$

In the paper quoted above it has been proved that the bias of an estimate of the risk premium, if $q$ is determined in the way suggested above, is of a smaller order of magnitude than the bias of an estimate using other rules for the determination of $q$.

6. In order to generalize the results of section 5 we will at first remove the assumption that the ceding company has only one elementary group but retain the assumption that ${ }_{c} R_{i t}^{\prime}$ for every $i$ is constantly equal to ${ }_{c} R_{i}^{\prime}$ independently of $t$. Then ${ }_{c} X_{t}$ is attached to an ergodic process. Writing $\left.c_{c} m^{*},{ }_{c} \sigma^{*}\right)^{2}$ and ${ }_{c} a_{v}^{*}$ for the first two semiinvariants and for the coefficients of the terms under the summation sign of the Edgeworth's expansion, all referring to ${ }_{c} X_{t}$, the risk premium payable by the company for a reinsurance according to section I covering all elementary groups as a whole can be deduced in the same way as in section 5 . In fact, the expression for this risk premium takes the same form as (3) with the substitution of ${ }_{c} \sigma^{*},{ }_{c} a_{v}^{*}$ and ${ }_{c} v_{Q}^{*}=$ $\frac{c^{*}-Q}{{ }_{c} \sigma^{*}}$ for $\sigma, a_{0}$ and $v_{q}$ respectively. 
By (2), writing $m_{i}, \lambda_{i j}$ for the mean and second order central moments of $y_{i}, c m^{*}$ can be obtained by a linear transformation of ${ }_{c} R_{i}^{\prime}$ with matrix $\left\{m_{i}\right\}$ and $\left({ }_{c} \sigma^{*}\right)^{2}$ as a quadratic form in the variables ${ }_{c} R_{i}^{\prime}$ with matrix $\left\{\lambda_{i j}\right\}$. The moments of the 3 rd and the $4^{\text {th }}$ order are related by analogous expressions of the 3 rd and the $4^{\text {th }}$ degree respectively to the corresponding moments of $y_{i}$.

If the variables $y_{i}$ are mutually correlated, the factor $\delta_{i}$ for a particular value of $i$ is dependent on the quotient between $\sigma_{i}$ and ${ }_{c} \sigma^{*}$. It was, however, found in the paper quoted above that the variation of $\delta_{i}$ within a large interval of this quotient was of a very small order of magnitude as compared with $r$. The average of $\delta_{i}$ for such an interval was, therefore, applied to the interval with a neglectable error. If such an average appropriate to the ceding company $c$ is denoted by ${ }_{c} \delta_{i}^{\prime}$ and the central moments of the second order of $\delta_{i}^{\prime} y_{i}$ and $\delta_{j}^{\prime} y_{j}$ are denoted by $\lambda_{i j}^{\prime}$, we shall define the square of the modified standard deviation, $\left[{ }_{0} \sigma^{* \prime}\right]^{2}$ by the quadratic form in the variables ${ }_{c} R_{i}^{\prime}$ with matrix $\left\{{ }_{c} \lambda_{i j}^{\prime}\right\}$. The risk premium of the reinsurance with $Q=m+k_{.} \sigma{ }^{* \prime}$ can then be estimated with ${ }_{c} \sigma^{* \prime} . K$ i.e. of the same form as given in (4). This formula is, thus, generalized to companies with several elementary groups where for all $i_{c} R_{i t}^{\prime}$ are constant, independently of $t$.

7. It remains to treat the general case of ${ }_{c} X_{t}$ according to (2) with ${ }_{c} R_{i t}^{\prime}$ dependent on $t$. Consider a time series of ${ }_{c} X_{t}$. One particular value of the time series, say for $t=t_{0}$, can be regarded as one particular value of a sample function pertaining to an ergodic process of the type treated in 6 with ${ }_{c} R_{i t}^{\prime}={ }_{c} R_{i t_{0}}^{\prime}$ independently of $t$. This leads to a risk premium payable for the period $t=t_{0}$ by the ceding company $c$ for a reinsurance covering for this period all elementary groups as a whole, which might be expressed in the form of (3). In fact, this risk premium equals the right member of (3) with the substitution of ${ }_{c} \sigma_{t_{0}}^{*}, c_{c} a_{0 t_{0}}$ and $c^{v} Q_{\tau_{0}}=\frac{{ }_{c} m_{t_{0}}^{*}-Q}{{ }_{c} \sigma_{t_{0}}^{*}}$ for $\sigma, a_{v}$ and $v_{q}$. This substitution involves that the index of the same quantities in the formula (3) applied to the case treated in 6. shall be extended to include $t_{0}$.

In the same way we can get a simplified formula for the general case as has been described in the last part of the previous section, by attaching the index $t_{0}$ to all statistics mentioned there. 
8. It shall be observed that if $Q$ is defined by a function of a quantity which depends on $p_{i t}$, as was the condition for the use of the simplified formula for the risk premium according to section 7 , and consequently varying with time as $z_{i i}$, the reinsurance will not equalize the variation concerned, e.g. if the money value is depreciated this might result in changes of ${ }_{c} X_{t}$ not accounted for in the changes of ${ }_{c} R_{i t}$ due to the same cause. If the reinsurance shall equalize even the effects on $Q$ of such changes the limit must be independent of any quantity depending on $t$. The same consequence will, for example, follow if $Q$ is determined as a constant fraction of the direct premium income of the ceding company. In fact, an increase of the risk shall, in principle, lead to raised tariff rates for computation of the direct premium. A rule of fixing $Q$ in constant proportion to a quantity related to ${ }_{c} R_{i t}^{\prime}$ cannot, therefore, be judged as disadvantageous to the idea of the reinsurance.

9. For the purpose of the actual computation of the risk premium to be paid by the company $c$ for a given accounting period $t_{0}$ it is necessary to know the risk sum distribution ${ }_{c} R_{i t_{0}}$ for that particular company, all other necessary estimates being independent of $c$.

Besides $p_{i t}$ other necessary estimates are either the mean, the central moments of the second order of $y_{i}$ and the directly estimated risk premium rate based on the sample mean of indemnities, or the mean and the central moments of the second to the fourth order of $y_{i}$. These values can be estimated from a time series of the total amount of claims grouped into elementary groups in a greater material than from only one ceding company, e.g. the material of all such companies reinsured with the same reinsurer.

For each elementary group of the last mentioned material $p_{i t}$ can be studied according to an ordinary method of regression analysis. Thereinafter, the semi-invariants of $y_{i}$ may be estimated after an appropriate trend-elimination in $z_{i t}$. For the study of the method of simplification, described above, $\boldsymbol{\delta}_{i}$ can be calculated from the same time series by estimating, with trend-elimination, the risk premium rates of each elementary group directly, i.e. based on the sample mean of indemnities, according to the method described in section 5 . For the calculation of the values ${ }_{c} \delta_{i}^{\prime}$, such values shall be calculated for different values of the quotient $\sigma_{i}$ to ${ }_{c} \sigma^{*}$, where the 
latter shall be based on different model-distributions of ${ }_{c} R_{i}$. After analyzing the results the model-distributions can be classified into classes for averaging $\delta_{i}$ giving the values of ${ }_{c} \delta_{i}^{\prime}$ for corresponding intervals of the ratio of $\sigma_{i}$ to ${ }_{c} \sigma^{*}$.

The practical computation of the risk premium for each $c$ and $t=t_{0}$ can then be accomplished in the following way.

${ }_{c} R_{i t_{0}}$ are multiplied with $p_{i t_{0}}$ to give ${ }_{c} R_{i t_{0}}^{\prime}$. The estimate of $p_{i t_{0}}$ could be based on the regression analysis referred to above and, eventually, corrected for events having occurred after the analysis.

As the first alternative the risk premium shall be calculated according to the simplified method described in the last part of section 6 which involves the multiplications of $\lambda_{i j}$ by the appropriate values of ${ }_{c} \delta_{i t_{0}}^{\prime}$ and $\delta_{c}^{\prime} \delta_{t_{0}}$ and the computation of ${ }_{c} m_{t_{0}}^{*}$ and ${ }_{c} \sigma_{t_{0}}^{*^{\prime}}$. The risk premium is then equal to $K_{c} \sigma_{t_{0}}^{*^{\prime}}$ for $Q^{\prime}=m+k_{c} \sigma_{t 0}^{*^{\prime}}$.

As another alternative the risk premium shall be based on a formula of the same form as that given in (3), which involves the calculations of ${ }_{c} m_{t_{0}}^{*},{ }_{c} \sigma_{t_{0}}^{*}$ and ${ }_{c} a_{v}^{*} t_{0}$. The risk premium is then given by (3) with the substitution of these quantities for $m, \sigma, a_{v}$ and of $Q$, determined according to some rule, for $q$. 